\title{
Tratamentos térmicos para a superação de dormência em sementes de uma espécie arbórea brasileira
}

\author{
Bruna Rafaella Ferreira Silva ${ }^{\mathrm{a}^{*}} \oplus$, Fernanda Moura Fonseca Lucas ${ }^{\mathrm{b}} \oplus$, Thalles Luiz Negreiros da Costa ${ }^{\mathrm{c}}$, \\ Tatiane Kelly Barbosa de Azevêdocio
}

${ }^{\text {a } U n i v e r s i d a d e ~ F e d e r a l ~ d e ~ L a v r a s, ~ B r a s i l ~}$

${ }^{\mathrm{b}}$ Universidade Federal do Paraná, Brasil

${ }^{\mathrm{c}}$ Universidade Federal do Rio Grande do Norte, Brasil

*Autor correspondente (brunarafaellaf@hotmail.com)

\section{N F O}

\section{Keywords}

impermeability of the

in tegument

timbaúva

germination

native species

\section{Palavras-chaves}

impermeabilidade do tegumento timbaúva

germinação espécie nativa

\begin{abstract}
A B S T R A C T
Heat treatments to overcome dormancy in seeds of a Brazilian tree species

Enterolobium contortisiliquum (Vell.) Morong., known as tamboril, is a promising species in the recovery of degraded areas. However, it presents integument dormancy in its seeds and it is necessary to find methods to overcome it. Therefore, the present study aimed to evaluate the dry heat efficiency on the viability and vigor of $E$. contortisiliquum seeds, as an alternative and practical method for overcoming dormancy. The experiment was conducted in the house of vegetation of the Specialized Agricultural Sciences Academic Unit of the Federal University of Rio Grande do Norte. The fruits, obtained from different matrices located in the municipality of Macaíba-RN, were manually processed. The heat treatments obtained through a drying oven during five minutes used: 60 (T1), $80(\mathrm{~T} 2)$, and $105^{\circ} \mathrm{C}(\mathrm{T} 3)$, the mechanical scarification (T0) of the seeds were used as a mediating treatment of the efficiency of the others. The emergency, first emergency count, and the emergency speed index were evaluated. Observing the results obtained, the best performance corresponded to the scarified seeds. The thermal treatments in dry heat were not efficient to overcome the dormancy of $E$. contortisiliquum seeds when compared with the traditional method of mechanical scarification. However, the methodology presents practicality in its execution which can be considered advantageous. Therefore, new studies with different combinations of time and temperature for the species are encouraged.
\end{abstract}




\section{INTRODUÇÃO}

O Enterolobium contortisiliquum (Vell.) Morong., popularmente conhecido como timbaúba ou tamboril, pertencente à Família Fabaceae, é uma árvore endêmica do Brasil, podendo ser encontrada nas regiões Nordeste, Centro-oeste, Sul e Sudeste associada aos biomas Caatinga, Cerrado e Mata Atlântica (Rodrigues et al., 2020). É uma espécie promissora na recuperação de áreas degradadas, por desenvolver um rápido crescimento inicial e por apresentar potencial para uso no método de regeneração direta em campos abandonados, como também na fitorremediação, sendo tolerante a solos contaminados por zinco e cobre (Meneghello e Mattei, 2004; Araujo e Paiva Sobrinho, 2011; Silva et al., 2011; Silva et al., 2018).

Um fator indispensável para a produção de mudas é conhecer o seu melhor método de propagação. No caso da propagação via sementes, a produção ganha estímulo quando se compreende possíveis impasses que venham a comprometer o desenvolvimento dos embriões. Lêdo (1977), estudando a causa da dormência de sementes de E. contortisiliquum, não observou a presença de inibidores de germinação nos embriões ou estruturas circundantes, concluindo assim que a dormência é devido à impermeabilidade do tegumento à água, o que é bastante comum em várias outras espécies florestais da família Fabaceae, como Delonix regia (Oliveira et al., 2018), Erythrina crista-galli L. (Mello et al., 2016), Schizolobium parahyba (Vellozo) S. F. Blake (Candido e Oliveira, 2018) e Adenanthera pavonina L. (Silva et al., 2020).

O entendimento dos processos germinativos, sobretudo daquelas sementes com dormência tegumentar, pode oferecer subsídios para a produção de mudas e recomposição de áreas degradadas. Segundo Pinã-Rodrigues et al. (2007), a dormência é considerada uma estratégia de perpetuação da espécie, principalmente daquelas em estágio inicial da sucessão ecológica. Entretanto, a dormência passa a ser um empecilho fora do meio natural, pois esse mecanismo faz com que a germinação ocorra de forma desuniforme, atrapalhando principalmente a produção de mudas, além de sujeitar as sementes a condições adversas, como o ataque de fungos, podendo torná-las inviáveis (Borges et al., 1982).

Estudos relacionados a métodos de superação de dormência física principalmente aqueles baseados na promoção de fissuras no tegumento, são importantes para permitir a entrada de água e gases pelos tecidos embrionários (Farias et al., 2019). Estes métodos podem ser de origem: i) térmica, como a inserção em água quente; ii) química, como a inserção em ácido sulfúrico; e iii) física ou mecânica, por meio fricção em material áspero (Picolotto et al., 2013; Muller et al., 2016).

Um dos métodos térmicos é inserção de sementes ao calor seco por meio do uso de estufas (Wutke et al., 1995). Estudos recentes têm testado essa metodologia, como um modelo alternativo aos processos convencionais, por apresentar-se vantajoso devido à praticidade e baixo risco (Castro, 2010; Almeida et al., 2018). Desse modo, o presente trabalho teve como objetivo avaliar a eficiência do calor seco sobre a viabilidade e o vigor das sementes de E. contortisiliquum.

\section{MATERIAL E MÉTODOS}

O experimento foi conduzido em casa de vegetação localizada no campus de Macaíba pertencente à Unidade Acadêmica Especializada em Ciências Agrárias da Universidade Federal do Rio Grande do Norte $\left(5^{\circ} 53^{\prime} 04.5^{\prime \prime S} 35^{\circ} 21^{\prime} 56.6^{\prime \prime} \mathrm{W}\right)$. Os frutos foram coletados no mesmo campus, da copa de duas matrizes distantes aproximadamente $1 \mathrm{~km}$ entre si. Em seguida, procedeu-se beneficiamento manual das sementes descartando as que apresentavam injurias ou deformações.

Reconhece-se que a escarificação mecânica é um método eficiente para superação da dormência do E. contortisiliquum, sendo recomendada por diversos trabalhos (Malavasi e Malavasi, 2004; Silva e Rosa, 2012). Deste modo, ela foi utilizada como parâmetro de eficiência para o experimento (T0), em que foi observado o desempenho dos seguintes tratamentos térmicos obtidos por meio de estufa de secagem (calor seco) durante cinco minutos: 60 (T1), 80 (T2) e $105^{\circ} \mathrm{C}(\mathrm{T} 3)$, totalizando quatro tratamentos.

Em seguida, realizou-se o teste de emergência (E), em que as sementes foram distribuídas em bandejas de poliestireno (isopor) contendo areia, a uma profundidade de $1,0 \mathrm{~cm}$, com irrigações diárias. Após a semeadura, as bandejas foram postas em casa de vegetação. No $30^{\circ}$ dia após a semeadura, foi computado o número de plântulas normais (Brasil, 2013) emersas, cujos resultados foram expressos em porcentagem. A primeira contagem de emergência (PC) foi conduzida conjuntamente ao teste de emergência e realizado no $8^{\circ}$ dia após a semeadura, computando-se à porcentagem de plântulas normais emersas. Também se avaliou o índice de velocidade de emergência (IVE) calculado conforme equação proposta por Maguire (1962).

$\mathrm{O}$ delineamento experimental utilizado foi o inteiramente ao acaso, constando de quatro tratamentos, com cinco repetições de 10 sementes cada. Os dados foram submetidos à análise de variância e as médias comparadas pelo teste de Tukey, a $5 \%$ de probabilidade, através do programa estatístico BioEstat 5.0. 


\section{RESULTADOS E DISCUSSÃO}

Os resultados do teste estatístico ANOVA, demonstraram que houve diferença significativa $(\mathrm{p}<$ $0.05)$ para todas as variáveis avaliadas (Tabela 1 ).

Tabela 1 - Taxa de emergência (E), primeira contagem (PC) e índice de velocidade de emergência (IVE) de sementes de Enterolobium contortisiliquum submetidas a diferentes tratamentos térmicos.

\begin{tabular}{cccc}
\hline Tratamento & E (\%) & PC (\%) & IVE \\
\hline Escarificação mecânica & $80 \mathrm{a}$ & $26 \mathrm{a}$ & $4,55 \mathrm{a}$ \\
ar seco $60^{\circ} \mathrm{C}$ & $40 \mathrm{~b}$ & $4 \mathrm{~b}$ & $1,86 \mathrm{~b}$ \\
ar seco $80^{\circ} \mathrm{C}$ & $46 \mathrm{~b}$ & $6 \mathrm{~b}$ & $1,67 \mathrm{~b}$ \\
ar seco $105^{\circ} \mathrm{C}$ & $46 \mathrm{~b}$ & $6 \mathrm{~b}$ & $1,83 \mathrm{~b}$ \\
\hline
\end{tabular}

Médias seguidas de mesma letra, na coluna, não diferem estatisticamente entre si, pelo teste de Tukey a 5\% de significância.
A emergência estabilizou-se após o vigésimo quarto dia (Figura 1). Ao analisar os dados obtidos foi possível constatar que o melhor resultado correspondeu ao tratamento com escarificação mecânica, obtendo $80 \%$ da taxa de emergência de plântulas normais, sendo esse valor duas vezes superior aos resultados oriundos dos demais tratamentos.

Os tratamentos em calor seco, em todas as temperaturas se mostraram pouco eficientes, obtendose menos de $50 \%$ de plântulas normais, além de não diferirem estatisticamente entre si, nas três variáveis observadas. Visava-se, em função das altas temperaturas, a possível dilatação do tegumento, causando assim, microfissuras. É possível que o baixo índice de taxa de emergência se deu ao curto tempo no qual as sementes foram submetidas ao calor, não sendo suficiente para promover o rompimento esperado.

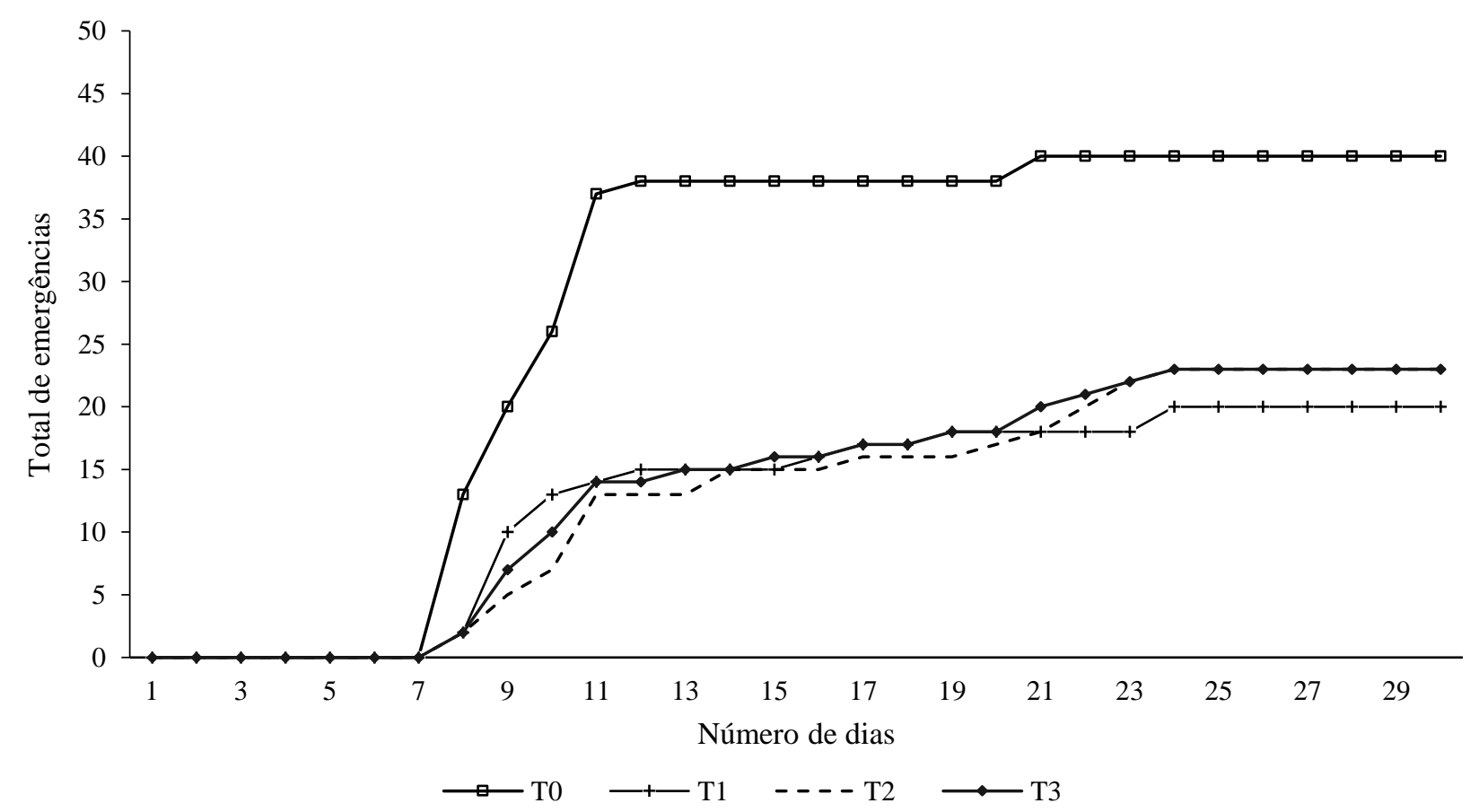

Figura 1 - Total acumulado de emergência das sementes de E. contortisiliquum submetidas aos quatro tratamentos: Controle (T0), $60^{\circ} \mathrm{C}(\mathrm{T} 1), 80^{\circ} \mathrm{C}(\mathrm{T} 2)$ e $105^{\circ} \mathrm{C}(\mathrm{T} 3)$, durante os 30 dias de avaliação.

Respostas semelhantes foram encontradas por Silva e Rosa (2012), em que a escarificação mecânica com lixa, foi o método mais eficiente para superação de dormência tegumentar em sementes de E. contortisiliquum quando comparado a utilização do calor seco a $65^{\circ} \mathrm{C}$ por mais de 12 horas. Almeida et al. (2018) também não apresentaram resultados satisfatórios com a utilização do calor seco para a espécie, onde a temperatura de $60^{\circ} \mathrm{C}$ com exposições entre 5 a 20 minutos não apresentaram diferenças, além de obter uma taxa de germinação inferior a $20 \%$, no entanto estes resultados podem ter sido influenciados pela qualidade do lote utilizado.

Métodos como escarificação mecânica e química podem ser utilizados para facilitar a germinação, simulando processos que ocorrem naturalmente no ambiente, a exemplo do percurso da semente no intestino de aves e mamíferos, assim como, a inserção a altas temperaturas pode simular 
a exposição ao sol como também a passagem de fogo, o qual permite o embrião contato com água e outros gases por meio do tegumento enfraquecido.

As médias obtidas pelos tratamentos neste estudo ainda foram superiores às encontradas por Parreira et al. (2012), no qual estudaram a superação de dormência de Momordica charantia L. quando expostas ao calor seco $\left(60^{\circ} \mathrm{C}\right)$ por 15 minutos, em que acarretou uma germinação inferior a $25 \%$, enquanto neste estudo, para a mesma temperatura, essa germinação foi de $40 \%$. Esse resultado mostra que a espécie E. contortisiliquum provavelmente possui maior afinidade com este tipo de superação de dormência.

Estudos relatam a aprovação do calor seco para superação de outras espécies, Alencar et al. (2009) obtiveram como resultado melhor eficiência na superação de dormência através do uso de temperaturas elevadas quando as sementes de Stylosanthes macrocephala e Stylosanthes capitata foram submetidas à temperatura de 60 e $70^{\circ} \mathrm{C}$, por 15 horas, respectivamente.Enquanto que no estudo de Carneiro et al. (1982) de Mimosa scabrella Benth. em tratamento térmico com calor seco de $60^{\circ} \mathrm{C}$ com exposições de 1 a 10 minutos, observou que a porcentagem de germinação cresceu de $40 \%$ (sem superação de dormência) pra 70\%, não havendo diferença no tempo de exposição.

Com relação à primeira contagem de emergência (PC), o tratamento em que se utilizou a escarificação mecânica como método de superação de dormência apresentou o maior percentual (26\%), valor bastante superior aos obtidos pelos demais tratamentos. Guedes et al. (2013) estudando formas de superar a dormência de sementes de Cassia fistula L., visualizaram que a maior porcentagem da primeira contagem de germinação foi no tratamento utilizando a escarificação mecânica com lixa $(63 \%)$, mostrando resultado semelhante ao encontrado neste trabalho.

Na Figura 2 é possível observar o índice de velocidade de emergência das sementes de E. contortisiliquum em função dos dias observados, tornando-se claro a eficiência do tratamento com escarificação mecânica quando comparado aos tratamentos com calor seco.

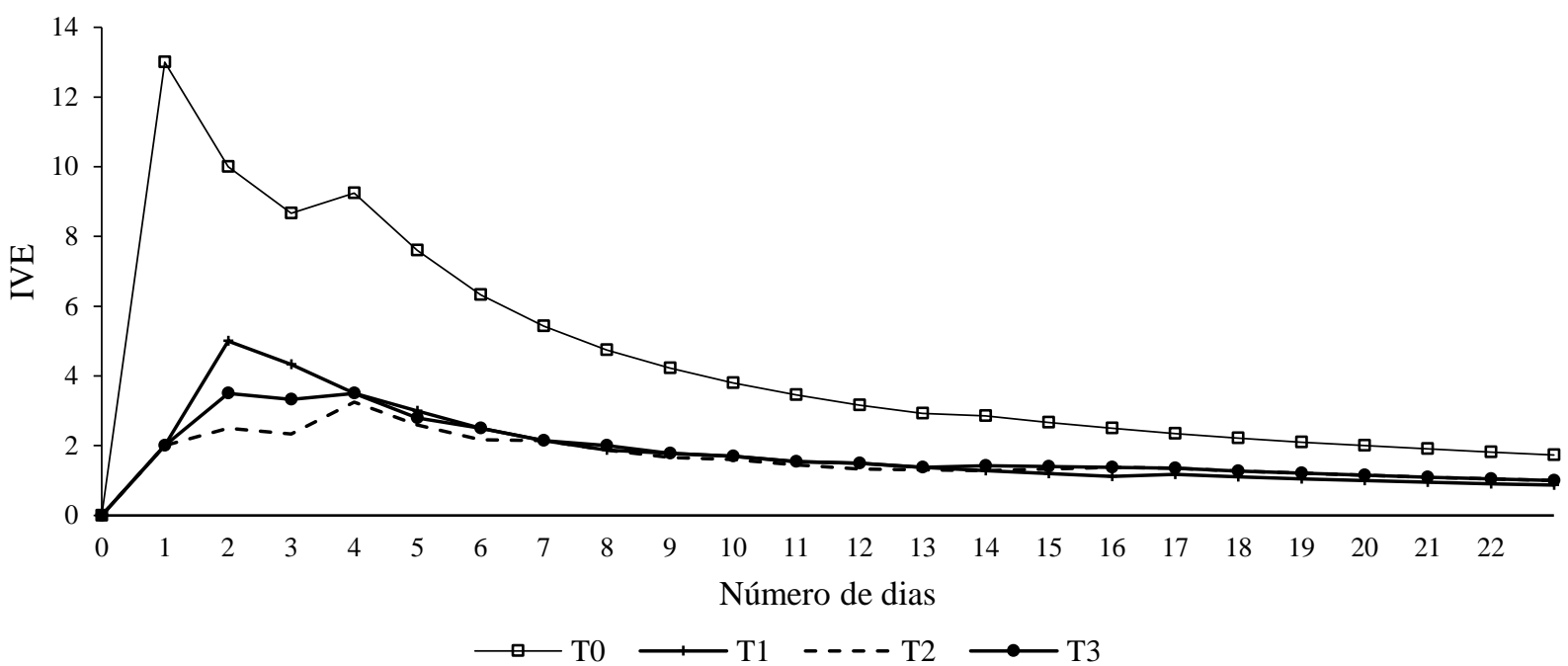

Figura 2 - Índice de velocidade de emergência das sementes de $E$. contortisiliquum submetidas aos quatro tratamentos: Controle (T0), $60^{\circ} \mathrm{C}(\mathrm{T} 1), 80^{\circ} \mathrm{C}(\mathrm{T} 2)$ e $105^{\circ} \mathrm{C}(\mathrm{T} 3)$, calculados após a primeira emergência.

Em estudo realizado por Ribeiro et al. (2017), no qual testaram métodos sustentáveis de superação de dormência em sementes de Hyminaea stigonocarpa Mart. ex. Hayne, o índice de velocidade de emergência também foi maior no tratamento utilizando a escarificação mecânica com lixa, quando comparada aos tratamentos utilizando altas temperaturas. Esse mesmo padrão foi visualizado por Lima et al. (2013) e Guedes et al. (2013) na superação de dormência das sementes de Delonix regia (Bojer ex. Hook.) Raf. e Cassia fistula, respectivamente. Tais resultados mostram que a escarificação mecânica é um dos métodos mais eficientes na superação de dormência das sementes de diversas espécies que possuem tegumento impermeável.

Apesar do resultado, é possível que os tratamentos térmicos demonstrem vantagens sobre a escarificação mecânica devido à praticidade para execução, principalmente quando há grandes quantidades de sementes, no entanto, para o E. contortisiliquum outros tratamentos devem ser testados. Conforme Eira et al. (1993), cada um dos métodos de superação apresenta pontos positivos e negativos, de modo que a metodologia deva ser analisada como um conjunto envolvendo além dos resultados, o custo e a praticidade. 


\section{CONCLUSÕES}

Os tratamentos térmicos em calor seco utilizando 60,80 e $105^{\circ} \mathrm{C}$ por 5 minutos não foram eficientes para superação da dormência das sementes de Enterolobium contortisiliquum quando comparado com o método de escarificação mecânica. Entretanto, a metodologia apresenta praticidade em sua execução o que pode ser considerado vantajoso. Sendo assim, incentiva-se a realização de novos estudos com diferentes combinações de tempo e temperatura para a espécie.

\section{REFERÊNCIAS BIBLIOGRÁFICAS}

Alexandre RS, Gonçalves FG, Rocha AP, Arruda MP, Lemes EP. Tratamentos físicos e químicos na superação de dormência em sementes de Enterolobium contortisiliquum (Vell.) Morong. Revista Brasileira de Ciências Agrárias, v.4, n.2, p.156-159, 2009. https://doi.org/10.5039/agraria.v $4 \mathrm{i} 2 \mathrm{a} 6$

Alencar KMC, Laura VA, Rodrigues APDC, Resende RMS. Tratamento térmico para superação da dormência em sementes de StylosanthesSW. (Fabaceae Papilionoideae). Revista Brasileira de Sementes, v.31, n.2, p.164-170, 2009. https://doi.org/10.1590/S0101-31222009000200019

Almeida DM, Ucella Filho JGM, Marques SRR, Azevedo TKB. Superação da dormência de sementes de Enterolobiumcontortisiliquum (Vell.) Morong. com utilização de calor seco. Agropecuária Científica no Semiárido, v.14, n.2, 2018. https://doi.org/10.30969/acsa.v14i2.989

Araujo AP, Paiva Sobrinho S. Germinação e produção de mudas de tamboril (Enterolobium contortisiliquum (vell.) Morong) em diferentes substratos. Revista Árvore, v.35, n.3, p.581-588, 2011. https://doi.org/10.1590/S010067622011000400001 .

Borges EEL, Borges RCGB, Candido JF, Gomes JM. Comparação de métodos de quebra de dormência em sementes de copaíba. Revista brasileira de sementes, v.4, n.01, p.912, 1982. https://doi.org/10.17801/0101-3122/rbs.v4n1p912

Brasil. Ministério da Agricultura, Pecuária e Abastecimento. Instruções para a análise de sementes de espécies florestais. Ministério da Agricultura, Pecuária e Abastecimento. Secretaria de Defesa Agropecuária. Brasília: Mapa/ACS, 2013. 98p.

Candido DF, Oliveira AS. Métodos para a superação da dormência em sementes de Schizolobiumparahyba (Vellozo) S. F. Blake (Fabaceae). Acta Biológica Catarinense, v.5, n.2, p.98-105, 2018. https://doi.org/10.21726/abc.v5i2.451

Carneiro RM, Almeida Jr AR, Kageyama PY, Dias IS. Importância da dormência das sementes na regeneração da Bracatinga - Mimosa scabrellaBenth. Instituto de Pesquisas e Estudos Florestais, n.149, 1982.

Castro DS. Superação de dormência em sementes de garapa (Apuleialeiocarpa (Vogel) J. F. Macbr.). 2010. 63p. Dissertação (Mestrado em Fitotecnia), Universidade Federal de Viçosa, Viçosa - MG.
Eira MRS, Freitas RWA, Mello CMC. Superação da dormência de sementes de Enterlobiumcontortisiliquum (Vell.) Morong. - Leguminosae. Revista Brasileira de Sementes, v.15, p.177-182, 1993.

https://doi.org/10.30969/acsa.v14i2.989

Farias CCM, Lopes JC, Mengarda LHG, Maciel KS, Moraes CE. Biometria, características físicas e absorção de água de sementes de EnterolobiummaximumDucke, Ciência Florestal, v.29, n.3, p.1241-1253, 2019.

https://doi.org/10.5902/1980509814887

Guedes RS, Alves EU, Santos-Moura SS, Costa EG, Melo PAFR. Tratamentos para superar dormência de sementes de Cassia fistula L. Biotemas, v.26, n.4, p.11-22, 2013. https://doi.org/10.5007/2175-7925.2013v26n4p11

Lêdo AAM. Estudo da causa da dormência em sementes de guapuruvu (Shizolobiumparahybum (Vell.) Blake e orelhade-negro (Enterolobiumcortortisiliquum (Vell.) Morong e métodos para sua quebra. 1997. 57p. (Dissertação de mestrado), Universidade Federal de Viçosa, Viçosa-MG.

Lima JS, Chaves AP, Medeiros MA, Rodrigues GSO, Benedito CP. Métodos de superação de dormência em sementes de flamboyant (Delonix regia). Revista verde, v.8, n.1, p.104-109, 2013.

Lorenzi H. Árvores brasileiras: Manual de identificação e cultivo de plantas arbóreas nativas do brasil. 4.ed. Nova Odessa: Instituto Plantarum, 384p. 2002.

Malavasi UC, Malavasi MM.Dormancy breaking and germination of Enterolobium contortisiliquum (Vell.) Morong seed. Brazilian Archives of Biology and Technology, v.47, n.6, 2004. http://dx.doi.org/10.1590/S151689132004000600003

Mello LM, Cantos AA, Meneghello GE, Villela FA. Superação de dormência e influência da temperatura, substrato e fotoperiodo na germinação de sementes de Erythrina crista-gali L. (Fabaceae). Revista Thema, v.13, n.3, p.3037, 2016. http://dx.doi.org/10.15536/thema.13.2016.3037.371

Meneghello GE, Mattei VL. Semeadura direta de Timbaúva (Enterolobiumcontortisiliquum), Canafistula (Peltophorumdubim) e Cedro (Cedrelafissilis), em campos abandonados. Ciência Florestal, Santa Maria, v.14, n.2, p. 21-27, 2004. https://doi.org/10.5902/198050981803

Muller EM, Gibbert P, Binotto T, Kaiser DK, Bartolini MF. Maturação e dormência em sementes de Peltophorumdubium (Spreng) Taub. de diferentes matrizes, Iheringia Série Botânica, v.71, n.3, p.222-229, 2016.

Oliveira KJB, Lima JSS, Andrade LIF, Costa JAMA, Crispim JF. Quebra de dormência de sementes de Delonix regia (Fabaceae). Revista de Ciências Agrárias, v. 41, n.3, p. 709-716, 2018. http://dx.doi.org/10.19084/RCA17302

Parreira MC, Cardozo NP, Pereira FCM, Alves PLCA. Superação de dormência das sementes e controle químico de Momordicacharantia L. Biosci. J., v.28, n.3, p.358-365, 2012.

Picolotto DRN, Theodoro JVC, Dias AR, Theodoro GF, Alves CZ. Germinação de sementes de urucum em função de métodos de superação de dormência e temperaturas. Pesquisa Agropecuária Tropical, v.43, n.3, p.232-238, 2013. https://doi.org/10.1590/S1983-40632013000300004 
Piña-Rodrigues FCM, Nogueira ES, Peixoto MC. Estado da arte da pesquisa em tecnologia de sementes de espécies florestais da Mata Atlântica. In: Piña-Rodrigues, FCM, Freire JM, Leles PSS, Breier TB. (org.). Parâmetros técnicos para produção de sementes florestais. Rede Mata Atlântica de Sementes Florestais. 1.ed. Seropédica: EDUR, 2007. Seropédica: UFRRJ, p.105-1141, 2007.

Ribeiro EA, Freitas GA, Freitas MABP, Santos ACM, Bessa NGF, Silva RR. Métodos sustentáveis para superação de dormência em sementes de Jatobá do Cerrado. Tecnologia e Ciência Agropecuária, v.11, n.6, p.119-124, 2017.

Rodrigues EM, Queiroz RT, Silva LP, Monteiro FKS, Melo JIM. Fabaceae em um afloramento rochoso no semiárido brasileiro. Rodriguésia, v.71, 2020. https://doi.org/10.1590/2175-7860202071025

Silva CTAC, Rosa APM. Tratamentos para superação da dormência em sementes de orelha-de-negro (Enterolobiumcontortisiliquum (Vell.) Morong.). Revista Varia Scientia Agrárias, v.2, n.2, p.79-90, 2012.

Silva GIN, Araújo ABN, Ferraz ACN, Silva CX, Silva MAD. Utilização de diferentes tratamentos pré-germinativos para superação de dormência em sementes de Adenantherapavonina L. Revista Diversitas, v.5, n.2, p.754-762, 2020. https://doi.org/10.17648/diversitas-journal-v5i2-1044

Silva RF, Ros CO, Antoniolli ZI, Grolli AL, Sheid DL, Bertollo GM, Missio EL. Crescimento e tolerância de mudas de Enterolobiumcontortisiliquumvell. Cultivadas em solo contaminado com zinco. Ciência Florestal, v.28, n.3, p.979-986, 2018

http://dx.doi.org/10.5902/1980509833374

Silva RF, Lupatini M, Antoniolli ZI, Leal LT, Moro Junior CA. Comportamento de Peltophorumdubium (Sprengel) Taubert, Parapiptadeniarigida (Bentham) Brenan e Enterolobiumcontortisiliquum (Vell.) Morong Cultivadas em Solo Contaminado com Cobre. Ciência Florestal, v.21, p.105-112, 2011. https://doi.org/10.5902/198050982752

Wutke EB, Maeda JA, Pio RM. Superação da dormência de sementes de mucuna-preta pela utilização de "calor seco". ScientiaAgricola, v.52, n.3, 1995. https://doi.org/10.1590/S0103-90161995000300013 